\title{
Axiomatizing Weak Ready Simulation Semantics over BCCSP *
}

\author{
Luca Aceto $^{1,3}$, David de Frutos Escrig, 2,3, Carlos Gregorio-Rodríguez ${ }^{2,3}$, and \\ Anna Ingolfsdottir ${ }^{1,3}$ \\ 1 ICE-TCS, School of Computer Science, Reykjavik University, Iceland \\ 2 Departamento de Sistemas Informáticos y Computación, \\ Universidad Complutense de Madrid, Spain \\ 3 Abel Extraordinary Chair (Universidad Complutense-Reykjavik University)
}

\begin{abstract}
Ready simulation has proven to be one of the most significant semantics in process theory. It is at the heart of a number of general results that pave the way to a comprehensive understanding of the spectrum of process semantics. Since its original definition by Bloom, Istrail and Meyer in 1995, several authors have proposed generalizations of ready simulation to deal with internal actions. However, a thorough study of the (non-)existence of finite (in)equational bases for weak ready simulation semantics is still missing in the literature. This paper presents a complete account of positive and negative results on the axiomatizability of weak ready simulation semantics over the language BCCSP. In addition, this study offers a thorough analysis of the axiomatizability properties of weak simulation semantics.
\end{abstract}

\section{Introduction}

Process algebras, such as ACP [9,11], CCS [33] and CSP [28], are prototype specification languages for reactive systems. Such languages offer a small, but expressive, collection of operators that can be combined to form terms that describe the behaviour of reactive systems.

Since the seminal work by Bergstra and Klop [11], and Hennessy and Milner [27], the search for (in)equational axiomatizations of notions of behavioural semantics for fragments of process algebras has been one of the classic topics of investigation within concurrency theory. A complete axiomatization of a

\footnotetext{
^ Luca Aceto and Anna Ingolfsdottir have been partially supported by the projects 'New Developments in Operational Semantics' (nr. 080039021) and 'Meta-theory of Algebraic Process Theories' (nr. 100014021) of the Icelandic Research Fund. David de Frutos Escrig and Carlos Gregorio-Rodríguez have been partially supported by the Spanish projects TESIS (TIN2009-14312-C02-01), DESAFIOS10 (TIN2009-14599-C0301) and PROMETIDOS S2009/TIC-1465. The paper was begun when David de Frutos Escrig and Carlos Gregorio-Rodríguez held Abel Extraordinary Chair positions at Reykjavik University, and finalized while Luca Aceto and Anna Ingolfsdottir held Abel Extraordinary Chairs at Universidad Complutense de Madrid, Spain, supported by the NILS Mobility Project.
} 
behavioural semantics yields a purely syntactic and model-independent characterization of the semantics of a process algebra, and paves the way to the application of theorem-proving techniques in establishing whether two process descriptions exhibit related behaviours.

There are three types of 'complete' axiomatizations that one meets in the literature on process algebras. An (in)equational axiomatization is called groundcomplete if it can prove all the valid (in)equivalences relating closed terms, i.e. terms with no occurrences of variables, in the process algebra of interest. It is complete when it can be used to derive all the valid (in)equivalences. (A complete axiom system is also referred to as a basis for the algebra it axiomatizes.) These two notions of completeness relate the semantic notion of process, namely an equivalence class of terms, with the proof-theoretic notion of provability from an (in)equational axiom system. An axiomatization $E$ is $\omega$-complete when an inequation can be derived from $E$ if, and only if, all of its closed instantiations can be derived from $E$. The notion of $\omega$-completeness is therefore a proof-theoretic one. Its connections with completeness are well known, and are discussed in, e.g., [4].

In [23], van Glabbeek studied the semantics in his spectrum in the setting of the process algebra BCCSP, which is sufficiently powerful to express all finite synchronization trees [33]. In the aforementioned reference, van Glabbeek gave, amongst a wealth of other results, (in)equational ground-complete axiomatizations for the preorders and equivalences in the spectrum. In [17], two of the authors of this paper presented a unification of the axiomatizations of all the semantics in the linear time-branching time spectrum. This unification is achieved by means of conditional axioms that provide a simple and clear picture of the similarities and differences between all the semantics. In [25], Groote obtained $\omega$-completeness results for most of the axiomatizations presented in [23], in case the alphabet of actions is infinite. The article [4] surveys results on the existence of finite, complete equational axiomatizations of equivalences over fragments of process algebras up to 2005. Some of the recent results on this topic may be found in $[5,6,8]$.

In the setting of BCCSP, in a seminal journal paper that collects and unifies the results in a series of conference articles, Chen, Fokkink, Luttik and Nain have offered in [15] a definitive classification of the status of the finite basis problem - that is, the problem of determining whether a behavioural equivalence has a finite, complete, equational axiomatization over the chosen process algebra-for all the semantics in van Glabbeek's spectrum. Notable later results by Chen and Fokkink presented in [14] give the first example of a semanticsthe so-called impossible future semantics from [40]—where the preorder defining the semantics can be finitely axiomatized over BCCSP, but its induced equivalence cannot. The authors of this paper have recently shown in [2] that complete simulation and ready simulation semantics do not afford a finite (in)equational axiomatization even when the set of actions is a singleton.

The collection of results mentioned in the previous paragraph gives a complete picture of the axiomatizability of behavioural semantics in van Glabbeek's 
spectrum over BCCSP. However, such notions of behavioural semantics are concrete, in the sense that they consider each action processes perform as being observable by their environment. Despite the fundamental role they play in the development of a theory of reactive systems, concrete semantics are not very useful from the point of view of applications. For this reason, notions of behavioural semantics that, in some well-defined way, abstract from externally unobservable steps of computation that processes perform have been proposed in the literature-see, e.g., the classic references [21,24,27], which offer, amongst many other results, ground-complete axiomatizations of the studied notion of behavioural semantics. Following Milner, such notions of behavioural semantics are usually called 'weak semantics' and the symbol $\tau$ is used to describe an unobservable action. However, to the best of our knowledge, no systematic study of the axiomatizability properties of variations on the classic notion of simulation semantics [31] that abstract away from internal steps of computation in the behaviour of processes has been presented in the literature. This is all the more surprising since simulation semantics is very natural and plays an important role in applications.

The aim of this paper is to offer a detailed study of the axiomatizability properties of the largest (pre)congruences over the language BCCSP induced by variations on the classic simulation preorder and equivalence that abstract from internal steps in process behaviours. We focus on the (pre)congruences associated with the weak simulation and the weak ready simulation $[12,29]$ preorders. For each of these behavioural semantics, we present results on the (non)existence of finite (ground-)complete (in)equational axiomatizations. Following [17], we also discuss the axiomatization of those semantics using conditional equations in some detail.

We begin our study of the weak simulation semantics over BCCSP in Section 3 by focusing on the natural extension of the classic simulation preorder to a setting with the internal action $\tau$. We show how to lift all the known results on the (non)existence of finite (ground-)complete axiomatizations from the setting of the classic simulation semantics to its weak counterpart using, for instance, the approach developed in [6].

In Section 4, we study the notion of weak ready simulation, namely a weak simulation that can only relate states that afford the same sets of observable actions. The finite axiomatizability of this semantics depends crucially on the presence of an infinite set of observable actions. Indeed, if the set of actions is infinite, we offer finite (un)conditional (ground-)complete axiomatizations for the weak ready simulation precongruence. In sharp contrast to this positive result, we prove that, when the set of observable actions $A$ is finite and nonempty, the (in)equational theory of the weak ready simulation precongruence over BCCSP does not have a finite (in)equational basis. 


\section{Preliminaries}

To set the stage for the developments offered in the rest of the paper, we present the syntax and the operational semantics for the language BCCSP, some background on (in)equational logic, and classic axiom systems for strong bisimulation equivalence and observational congruence [33].

Syntax of $B C C S P \operatorname{BCCSP}\left(A_{\tau}\right)$ is a basic process algebra for expressing finite process behaviour. Its syntax consists of closed (process) terms $p, q, r$ that are constructed from a constant $\mathbf{0}$, a binary operator _ $+_{-}$called alternative composition, or choice, and unary prefix operators $\alpha_{-}$, where $\alpha$ ranges over some set $A_{\tau}$ of actions of the form $A \cup\{\tau\}$, where $\tau$ is a distinguished action symbol that is not contained in $A$. Following Milner, we use $\tau$ to denote an internal, unobservable action of a reactive system, and we let $a, b, c$ denote typical elements of $A$ and $\alpha$ range over $A_{\tau}$. The set of closed terms is named $\mathrm{T}\left(\operatorname{BCCSP}\left(A_{\tau}\right)\right)$, in short $\mathrm{T}\left(A_{\tau}\right)$. We write $|A|$ for the cardinality of the set of observable actions.

Open terms $t, u, v$ can moreover contain occurrences of variables from a countably infinite set $V$ (with typical elements $x, y, z)$. We use $\mathbb{T}\left(\operatorname{BCCSP}\left(A_{\tau}\right)\right.$ ), in short $\mathbb{T}\left(A_{\tau}\right)$, to denote the set of open terms. The depth of a term $t$ is the maximum nesting of prefix operators in $t$.

In what follows, for each non-negative integer $n$ and term $t$, we use $a^{n} t$ to stand for $t$ when $n=0$, and for $a\left(a^{n-1} t\right)$ otherwise. As usual, trailing occurrences of $\mathbf{0}$ are omitted; for example, we shall usually write $\alpha$ in lieu of $\alpha \mathbf{0}$.

A (closed) substitution maps variables in $V$ to (closed) terms. For every term $t$ and substitution $\sigma$, the term $\sigma(t)$ is obtained by replacing every occurrence of a variable $x$ in $t$ by $\sigma(x)$. Note that $\sigma(t)$ is closed if $\sigma$ is a closed substitution.

Transitions and their defining rules Intuitively, closed $\operatorname{BCCSP}\left(A_{\tau}\right)$ terms represent finite process behaviours, where $\mathbf{0}$ does not exhibit any behaviour, $p+q$ is the nondeterministic choice between the behaviours of $p$ and $q$, and $\alpha p$ executes action $\alpha$ to transform into $p$. This intuition is captured, in the style of Plotkin [36], by the simple transition rules below, which give rise to $A_{\tau}$-labelled transitions between closed terms.

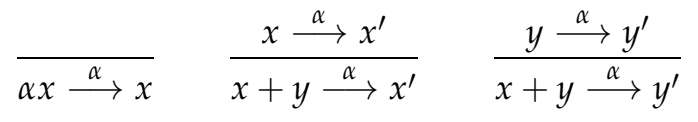

The operational semantics is extended to open terms by assuming that variables do not exhibit any behaviour.

The so-called weak transition relations $\stackrel{\alpha}{\Longrightarrow}\left(\alpha \in A_{\tau}\right)$ are defined over $\mathbb{T}\left(A_{\tau}\right)$ in the standard fashion as follows.

- We use $\stackrel{\tau}{\Longrightarrow}$ for the reflexive and transitive closure of $\stackrel{\tau}{\longrightarrow}$.

- For each $a \in A$ and for all terms $t, u \in \mathbb{T}\left(A_{\tau}\right)$, we have that $t \stackrel{a}{\Longrightarrow} u$ if, and only if, there are $t_{1}, t_{2} \in \mathbb{T}\left(A_{\tau}\right)$ such that $t \stackrel{\tau}{\Longrightarrow} t_{1} \stackrel{a}{\longrightarrow} t_{2} \stackrel{\tau}{\Longrightarrow} u$.

For each term $t$, we define $I^{*}(t)=\left\{a \mid a \in A\right.$ and $t \stackrel{a}{\Longrightarrow} t^{\prime}$ for some $\left.t^{\prime}\right\}$. 
Preorders and their kernels We recall that a preorder $\precsim$ is a reflexive and transitive relation. In what follows, any preorder $\precsim$ we consider will first be defined over the set of closed terms $\mathrm{T}\left(A_{\tau}\right)$. For terms $t, u \in \mathbb{T}\left(A_{\tau}\right)$, we define $t \precsim u$ if, and only if, $\sigma(t) \precsim \sigma(u)$ for each closed substitution $\sigma$.

The kernel $\approx$ of a preorder $\approx$ is the equivalence relation it induces, and is defined thus: $t \approx u$ if, and only if, ( $t \precsim u$ and $u \precsim t$ ). It is easy to see that the kernel of a preorder $\precsim$ is the largest symmetric relation included in $\precsim$.

Inequational logic An inequation (respectively, an equation) over the language $\operatorname{BCCSP}\left(A_{\tau}\right)$ is a formula of the form $t \leq u$ (respectively, $t=u$ ), where $t$ and $u$ are terms in $\mathbb{T}\left(A_{\tau}\right)$. An (in)equational axiom system is a set of (in)equations over the language $\operatorname{BCCSP}\left(A_{\tau}\right)$. An equation $t=u$ is derivable from an equational axiom system $E$, written $E \vdash t=u$, if it can be proven from the axioms in $E$ using the rules of equational logic (viz. reflexivity, symmetry, transitivity, substitution and closure under $\operatorname{BCCSP}\left(A_{\tau}\right)$ contexts).

$$
t=t \quad \frac{t=u}{u=t} \quad \frac{t=u u=v}{t=v} \quad \frac{t=u}{\sigma(t)=\sigma(u)} \quad \frac{t=u}{\alpha t=\alpha u} \quad \frac{t=u t^{\prime}=u^{\prime}}{t+t^{\prime}=u+u^{\prime}}
$$

For the derivation of an inequation $t \leq u$ from an inequational axiom system $E$, the rule for symmetry-that is, the second rule above-is omitted. We write $E \vdash t \leq u$ if the inequation $t \leq u$ can be derived from $E$.

It is well known that, without loss of generality, one may assume that substitutions happen first in (in)equational proofs, i.e., that the fourth rule may only be used when its premise is one of the (in)equations in $E$. Moreover, by postulating that for each equation in $E$ also its symmetric counterpart is present in $E$, one may assume that applications of symmetry happen first in equational proofs, i.e., that the second rule is never used in equational proofs. (See, e.g., [15, page 497] for a thorough discussion of this notion of 'normalized equational proof.') In the remainder of this paper, we shall always tacitly assume that equational axiom systems are closed with respect to symmetry. Note that, with this assumption, there is no difference between the rules of inference of equational and inequational logic. In what follows, we shall consider an equation $t=u$ as a shorthand for the pair of inequations $t \leq u$ and $u \leq t$.

The depth of $t \leq u$ and $t=u$ is the maximum of the depths of $t$ and $u$. The depth of a collection of (in)equations is the supremum of the depths of its elements. So, the depth of a finite axiom system $E$ is zero, if $E$ is empty, and it is the largest depth of its (in)equations otherwise.

An inequation $t \leq u$ is sound with respect to a given preorder relation $\precsim$ if $t \precsim u$ holds. An (in)equational axiom system $E$ is sound with respect to $\precsim$ if so is each (in)equation in $E$.

Classic axiomatizations for notions of bisimilarity The well-known axioms $B_{1}-B_{4}$ for $\operatorname{BCCSP}\left(A_{\tau}\right)$ given below stem from [27]. They are $\omega$-complete [35], and sound and ground-complete $[27,33]$, over $\operatorname{BCCSP}\left(A_{\tau}\right)$ (over any nonempty set of actions) modulo bisimulation equivalence, which is the finest semantics in 
van Glabbeek's spectrum [23].

$$
\begin{array}{rlrl}
B_{1} & x+y & =y+x \\
B_{2} & (x+y)+z & =x+(y+z) \\
B_{3} & x+x & =x \\
B_{4} & x+\mathbf{0} & =x
\end{array}
$$

In what follows, for notational convenience, we consider terms up to the least congruence generated by axioms $B_{1}-B_{4}$, that is, up to bisimulation equivalence. We use summation $\sum_{i=1}^{n} t_{i}$ (with $n \geq 0$ ) to denote $t_{1}+\cdots+t_{n}$, where the empty sum denotes 0 . Modulo the equations $B_{1}-B_{4}$ each term $t \in \mathbb{T}\left(A_{\tau}\right)$ can be written in the form $\sum_{i=1}^{n} t_{i}$, where each $t_{i}$ is either a variable or is of the form $\alpha t^{\prime}$, for some action $\alpha$ and term $t^{\prime}$.

In a setting with internal transitions, the classic work of Hennessy and Milner on weak bisimulation equivalence and on the largest congruence included in it, observational congruence, shows that the axioms $B_{1}-B_{4}$ together with the axioms $W_{1}-W_{3}$ below are sound and complete over $\operatorname{BCCSP}\left(A_{\tau}\right)$ modulo observational equivalence. (See $[27,33,34]$.)

$$
\begin{aligned}
W_{1} & \alpha x & =\alpha \tau x \\
W_{2} & \tau x & =\tau x+x \\
W_{3} & \alpha(\tau x+y) & =\alpha(\tau x+y)+\alpha y
\end{aligned}
$$

The above axioms are often referred to as the $\tau$-laws. For ease of reference, we write

$$
B W=\left\{B_{1}, B_{2}, B_{3}, B_{4}, W_{1}, W_{2}, W_{3}\right\} .
$$

\section{Weak Simulation}

We begin our study of the equational theory of weak simulation semantics by considering the natural, $\tau$-abstracting version of the classic simulation preorder [31]. We start by defining the notion of weak simulation preorder and the equivalence relation it induces. We then argue that all the known positive and negative results on the existence of (ground-)complete (in)equational axiomatizations for the concrete simulation semantics over the language $\operatorname{BCCSP}\left(A_{\tau}\right)$ can be lifted to the corresponding weak semantics.

Definition 1. The weak simulation preorder, denoted by $\precsim_{S}$, is the largest relation over terms in $\mathrm{T}\left(A_{\tau}\right)$ satisfying the following condition whenever $p \precsim_{S} q$ and $\alpha \in A_{\tau}$ :

- if $p \stackrel{\alpha}{\longrightarrow} p^{\prime}$ then there exists some $q^{\prime}$ such that $q \stackrel{\alpha}{\Longrightarrow} q^{\prime}$ and $p^{\prime} \precsim_{S} q^{\prime}$.

We say that $p, q \in \mathrm{T}\left(A_{\tau}\right)$ are weak simulation equivalent, written $p \approx_{S} q$, iff $p$ and $q$ are related by the kernel of $\precsim_{s}$, that is when both $p \precsim_{s} q$ and $q \precsim_{s} p$ hold.

Unlike many other notions of behavioural relations that abstract away from internal steps in the behaviour of processes, see [24,33] for classic examples, the weak simulation preorder is a precongruence over the language we consider in this study. 
Proposition 1. The preorder $\precsim_{S}$ is a precongruence over $\mathrm{T}\left(A_{\tau}\right)$. Hence $\approx_{S}$ is a congruence over $\mathrm{T}\left(A_{\tau}\right)$. Moreover, the axiom

$$
(\tau e) \tau x=x
$$

holds over $\mathrm{T}\left(A_{\tau}\right)$ modulo $\approx_{S}$.

The soundness of equation $\tau e$ is the key to all the results on the equational theory of the weak simulation semantics we present in the remainder of this section. In establishing the negative results, we shall make use of the reduction technique from the paper [6].

\subsection{Ground-completeness for weak simulation}

Besides the equation $\tau e$ previously stated in Proposition 1, there will be another important equation to consider in order to achieve an axiomatic characterization of the weak simulation preorder, namely

$$
\text { (S) } x \leq x+y
$$

This equation also plays an essential role in the axiomatization of the simulation preorder in the concrete case $[18,23]$.

Proposition 2. The set of equations

$$
E_{S \leq}=\left\{B_{1}, B_{2}, B_{3}, B_{4}, S, \tau e\right\}
$$

is sound and ground-complete for $\operatorname{BCCSP}\left(A_{\tau}\right)$ modulo $\precsim s .{ }^{4}$

Note that the equations $W_{1}-W_{3}$, even if sound for $\precsim_{S}$, are not needed in order to obtain a ground-complete axiomatization of $\precsim_{S}$ over $\operatorname{BCCSP}\left(A_{\tau}\right)$. Those equations can easily be derived from the axiom system in Proposition 2.

To obtain an axiomatization for the weak simulation equivalence, we need the equation

$$
\text { (SE) } a(x+y)=a(x+y)+a y \quad(a \in A) .
$$

This equation is well known from the setting of standard simulation equivalence, where it is known to be the key to a ground-complete axiomatization [23].

Proposition 3. The set of equations

$$
E_{S}=\left\{B_{1}, B_{2}, B_{3}, B_{4}, S E, \tau e\right\}
$$

is sound and ground-complete for $\operatorname{BCCSP}\left(A_{\tau}\right)$ modulo $\approx_{S}$.

\footnotetext{
${ }^{4}$ This completeness result was announced in [39] by van Glabbeek.
} 


\section{$3.2 \omega$-completeness for weak simulation}

Propositions 2 and 3 offer ground-complete axiomatizations for the weak simulation preorder and its kernel over $\operatorname{BCCSP}\left(A_{\tau}\right)$. The inequational axiomatization of the weak simulation preorder is finite, and so is the one for its kernel if the set of actions $A$ is finite. In the presence of an infinite collection of actions, the axiom system in Proposition 3 is finite if we consider $a$ to be an action variable. It is natural to wonder whether the weak simulation semantics afford finite (in)equational axiomatizations that are complete over $\mathbb{T}\left(A_{\tau}\right)$. The following results answer this question.

Proposition 4. If the set of actions is infinite, then the axiom system

$$
E_{S \leq}=\left\{B_{1}, B_{2}, B_{3}, B_{4}, S, \tau e\right\}
$$

is both $\omega$-complete and complete over $\operatorname{BCCSP}\left(A_{\tau}\right)$ modulo $\precsim s$.

So the weak simulation preorder can be finitely axiomatized over $\mathbb{T}\left(A_{\tau}\right)$ when $A$ is infinite. This state of affairs changes dramatically when $A$ is a finite collection of actions of cardinality at least two.

Theorem 1. If $1<|A|<\infty$, then the weak simulation equivalence does not afford a finite equational axiomatization over $\mathbb{T}\left(A_{\tau}\right)$. In particular, no finite axiom system over $\mathbb{T}\left(A_{\tau}\right)$ that is sound modulo weak simulation equivalence can prove all the (valid) equations in the family on page 511 of [15].

Corollary 1. If $1<|A|<\infty$, then the weak simulation preorder does not afford a finite inequational axiomatization over $\mathbb{T}\left(A_{\tau}\right)$.

Remark 1. If $A$ is a singleton then the simulation preorder coincides with trace inclusion. In that case, the simulation preorder is finitely based over $\mathbb{T}(A)$, as is simulation equivalence - see, e.g., [4]. Those axiomatizations can be lifted to the setting of weak simulation semantics simply by adding the equation $\tau e$ to any complete axiomatization of the simulation preorder or equivalence.

\begin{tabular}{|c|c|c|c|c|}
\hline \multirow{2}{*}{$\begin{array}{c}\text { Weak Simulation } \\
\text { Finite Equations }\end{array}$} & \multicolumn{2}{|c|}{ Ground-complete } & \multicolumn{2}{c|}{ Complete } \\
\cline { 2 - 5 } & Order & Equiv. & Order & Equiv. \\
\hline$|A|=\infty$ & $E_{S \leq}$ & $E_{S=}$ & $E_{S \leq}$ & $E_{S=}$ \\
\hline $1<|A|<\infty$ & $E_{S \leq}$ & $E_{S=}$ & \multicolumn{2}{|c|}{ Do not exist } \\
\hline$|A|=1$ & $E_{S_{1}^{\leq}}$ & $E_{S_{1}^{=}}$ & $E_{S_{1}^{\leq}}$ & $E_{S_{1}^{=}}$ \\
\hline
\end{tabular}

Table 1. Axiomatizations for the weak simulation semantics 


\begin{tabular}{|c|c|}
\hline $\begin{array}{l}E_{S \leq}=\left\{B_{1}-B_{4}, \tau e, S\right\} \\
E_{S=}=\left\{B_{1}-B_{4}, \tau e, S E\right\} \\
E_{S_{1}^{\leq}}=\left\{B_{1}-B_{4}, \tau e, S, T E, S g \leq\right\} \\
E_{S_{1}^{=}}=\left\{B_{1}-B_{4}, \tau e, T E, S g\right\}\end{array}$ & $\begin{array}{ll}(\tau e) & \tau x=x \\
(S) & x \leq x+y \\
(S g \leq) & x \leq a x \\
(S E) & a(x+y)= \\
& \quad a(x+y)+a y \\
(T E) & a(x+y)=a x+a y \\
(S g) & a x=a x+x\end{array}$ \\
\hline
\end{tabular}

Table 2. Axioms for the weak simulation semantics

Tables 1 and 2 summarize the positive and negative results on the existence of finite axiomatizations for weak simulation semantics. On Table 1 , and in subsequent ones, 'Do not exist' indicates that there is no finite (in)equational axiomatization for the corresponding semantic relation.

\section{Weak Ready Simulation}

In this section, we shall study the equational theory of the largest precongruence included in the weak ready simulation preorder. We first define the notion of weak ready simulation that will be the cornerstone in subsequent developments. We then proceed to study its induced precongruence, first in the case in which the set of actions $A$ is infinite and then in case that $A$ is finite.

In order to define the weak ready simulation semantics we recall the definition of the function $I^{*}$, presented in Section 2, that returns the set of initial visible actions of a term: $I^{*}(t)=\left\{a \mid a \in A\right.$ and $t \stackrel{a}{\Longrightarrow} t^{\prime}$ for some $\left.t^{\prime}\right\}$.

Definition 2. The weak ready simulation preorder, which we denote by $\precsim_{R S}$, is the largest relation over terms in $\mathrm{T}\left(A_{\tau}\right)$ satisfying the following conditions whenever $p \precsim R S q$ and $\alpha \in A_{\tau}$ :

- if $p \stackrel{\alpha}{\longrightarrow} p^{\prime}$ then there exists some term $q^{\prime}$ such that $q \stackrel{\alpha}{\Longrightarrow} q^{\prime}$ and $p^{\prime} \precsim_{R S} q^{\prime}$, and

- $I^{*}(p)=I^{*}(q)$.

We say that $p, q \in \mathrm{T}\left(A_{\tau}\right)$ are weak ready simulation equivalent, written $p \approx_{R S} q$, iff $p$ and $q$ are related by the kernel of $\precsim_{R S}$, that is when both $p \precsim_{R S} q$ and $q \precsim_{R S} p$ hold.

Remark 2. $\precsim_{R S}$ is not a precongruence with respect to the choice operator of $\operatorname{BCCSP}\left(A_{\tau}\right)$. It is immediate to show that $\tau a \precsim R S a$. However, $\tau a+b \mathscr{L}_{R S} a+b$.

There are many possible ways to define a weak ready simulation semantics (see, for instance, [22]). The preorder defined above is based on a natural 'weak counterpart' of the constraint used in capturing ready simulation as a 
constrained simulation [18]. This design criterion was important for us, because we aim at extending the general and natural results we obtained for this family of semantics from the strong to the weak setting.

There are, of course, other guiding principles that could lead to alternative definitions. For instance, the inequation $\alpha x \leq \alpha x+\alpha y\left(\alpha \in A_{\tau}\right)$ appears in [16] as a benchmark for characterizing weak preorders. Although the authors of [16] do not claim to define weak ready simulation semantics, from an algebraic viewpoint it would be appealing to have a weak ready simulation preorder satisfying this inequation. Unfortunately, so far we have failed to obtain a simple operational characterization of a weak simulation satisfying the previous inequation, even allowing for the addition of some other (simple and reasonable) axioms. Another reasonable requirement one might want to impose on a notion of weak ready simulation preorder is that it be preserved by hiding. As our readers can easily check, the preorder in Definition 2 does not meet this requirement. The study of the (in)equational theory of the largest precongruence with respect to hiding included in the weak ready simulation preorder defined above is an interesting topic for future research.

Summing up, we do not claim that our weak ready simulation is 'the right

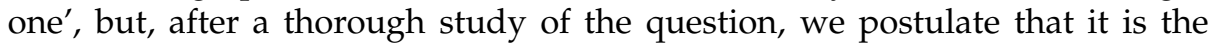
simplest notion of weak ready simulation that affords both a simple operational definition, as we have seen above, and good algebraic properties over BCCSP, as we will see below. Much more on the subject can be found in the extended version of the paper.

Definition 3. We denote by $\sqsubseteq_{R S}$ the largest precongruence included in $\precsim_{R S}$. Formally, $p \sqsubseteq_{R S} q$ iff

$-p \precsim_{R S} q$,

- $p \precsim_{R S} q \Rightarrow \forall \alpha \in A_{\tau} \quad \alpha p \precsim_{R S} \alpha q$, and

- $p \precsim_{R S} q \Rightarrow \forall r \in \mathrm{T}\left(A_{\tau}\right) \quad p+r \precsim_{R S} q+r$.

The behavioural characterization of the relation $\sqsubseteq_{R S}$ and its axiomatic properties will depend crucially on whether the set of visible actions $A$ is finite or infinite.

\subsection{Axiomatizing $\sqsubseteq_{R S}$ when $A$ is infinite}

If the set of actions $A$ is infinite, then we can obtain a behavioural characterization of the largest precongruence included in the weak ready simulation preorder using a standard construction due to Milner.

Definition 4. The preorder relation $\lesssim_{R S}$ between processes is defined as follows: We say that $p \lesssim_{R S} q$ iff

- for any $\alpha \in A_{\tau}$ such that $p \stackrel{\alpha}{\longrightarrow} p^{\prime}$, there exists some $q^{\prime}$ such that $q \stackrel{\tau}{\longrightarrow} \stackrel{\alpha}{\longrightarrow} \stackrel{\tau}{\longrightarrow} q^{\prime}$ with $p^{\prime} \precsim_{R S} q^{\prime}$, and

- $I^{*}(p)=I^{*}(q)$. 
We denote the kernel of $\lesssim_{R S}$ by $\bar{\sim}_{R S}$.

Proposition 5 (Behavioural characterization of $\sqsubseteq_{R S}$ ). If $A$ is infinite then $p \lesssim_{R S}$ $q$ if, and only if, $p \sqsubseteq_{R S} q$, for all $p, q \in \mathrm{T}\left(A_{\tau}\right)$. Therefore, $\bar{\sim}_{R S}$ coincides with the kernel of the preorder $\sqsubseteq_{R S}$.

Ground-completeness We shall now provide ground-complete (conditional) axiomatizations of the relations $\lesssim_{R S}$ and $\bar{\sim}_{R S}$.

To axiomatize $\lesssim_{R S}$ using conditional inequations, the key axiom is

$$
\left(R S_{\tau}\right) \quad I^{*}(x)=I^{*}(y) \Rightarrow x \leq x+y .
$$

This axiom mirrors the one used in the concrete setting in $[17,23]$.

Proposition 6. The set of equations

$$
E_{R S \leq}^{c}=B W \cup\left\{R S_{\tau}\right\},
$$

in which $R S_{\tau}$ is conditional, is sound and ground-complete for $\lesssim_{R S}$ over the language $\operatorname{BCCSP}\left(A_{\tau}\right)$.

We now give a ground-complete and unconditional axiomatization for the weak ready simulation preorder. For that we will consider the equations

$$
\begin{gathered}
(R S) \quad a x \leq a x+a y \\
(\tau g) \quad x \leq \tau x .
\end{gathered}
$$

Equation $R S$ is a well known and important one in the study of process semantics. $R S$ appears as a necessary condition for process semantics in many general results in process theory-see, e.g. [3, 19,20,30].

As for equation $\tau g$, this is indeed a simple and natural one that is satisfied by any 'natural' precongruence on processes with silent moves.

Theorem 2. The set of non-conditional equations defined by

$$
E_{R S} \leq=B W \cup\{R S, \tau g\}
$$

is sound and ground-complete for $\operatorname{BCCSP}\left(A_{\tau}\right)$ modulo $\lesssim_{R S}$.

To obtain a ground-complete axiomatization of the largest congruence included in weak ready simulation equivalence, it would be desirable to use a general 'ready-to-preorder result' $[3,20]$ as the one we have for the concrete case. There is indeed a similar result for weak semantics by Chen, Fokkink and van Glabbeek, see [16], but unfortunately it is not general enough to cover the case of the weak ready simulation congruence in Definition 4.

We now provide a ground-completeness result in which a key role is played by the equation

$$
\left(R S E_{\tau}\right) I^{*}(x)=I^{*}(y) \Rightarrow \alpha(x+y)=\alpha(x+y)+\alpha y,
$$

which is quite similar to the equation needed for the concrete case. 
Proposition 7. The set of equations

$$
E_{R S}^{c}=B W \cup\left\{R S E_{\tau}\right\},
$$

in which $R S E_{\tau}$ is conditional, is sound and ground-complete for $\bar{\sim}_{R S}$ over the language $\operatorname{BCCSP}\left(A_{\tau}\right)$.

In order to give an unconditional axiomatization of $\bar{\sim}_{R S}$, we consider the following equations:

$$
\begin{array}{ll}
(R S E) & \alpha(b x+z+b y)=\alpha(b x+z+b y)+\alpha(b x+z) \\
\left(R S E_{\tau e}\right) & \alpha(x+\tau y)=\alpha(x+\tau y)+\alpha(x+y) .
\end{array}
$$

Theorem 3. The set of equations

$$
E_{R S}=B W \cup\left\{R S E, R S E_{\tau e}\right\}
$$

is sound and ground-complete for $\operatorname{BCCSP}\left(A_{\tau}\right)$ modulo $\bar{\sim}_{R S}$.

$\omega$-completeness We shall now provide an axiomatization for the relation $\lesssim_{R S}$ that is $(\omega$-)complete.

Theorem 4. If the set of actions $A$ is infinite, then the axiom system

$$
E_{R S}=B W \cup\{R S, \tau g\}
$$

is both $\omega$-complete and complete for $\operatorname{BCCSP}\left(A_{\tau}\right)$ modulo $\lesssim_{R S}$.

\subsection{Axiomatizing $\sqsubseteq_{R S}$ when $A$ is finite}

Proposition 5 gives an explicit characterization of the largest precongruence included in the weak ready simulation preorder when the collection of actions is infinite. In this section, we shall study the (in)equational theory of $\sqsubseteq_{R S}$ when the set of observable actions $A$ is finite and non-empty.

First of all, note that if $A$ is finite then the relation $\lesssim_{R S}$ defined in Definition 4 is not the largest precongruence included in the weak ready simulation preorder. To see this, consider the terms

$$
p=\tau \sum_{a \in A} a \text { and } q=\sum_{a \in A} a .
$$

Observe that, for each $r \in \operatorname{BCCSP}\left(A_{\tau}\right)$, the following statements hold:

1. $p \precsim_{R S} q+r$ and

2. $p+r \precsim_{R S} q+r$.

From these statements if follows that $p \leq q$ is valid modulo the largest precongruence included in $\precsim_{R S}$. 
Definition 5. The relation $\lesssim_{R S}^{F}$ is defined as follows: We say that $p \lesssim_{R S}^{F} q$ iff

- for each $a \in A$ and $p^{\prime}$ such that $p \stackrel{a}{\longrightarrow} p^{\prime}$, there exists some $q^{\prime}$ such that $q \stackrel{a}{\longrightarrow} q^{\prime}$ with $p^{\prime} \precsim_{R S} q^{\prime}$;

- for each $p^{\prime}$ such that $p \stackrel{\tau}{\longrightarrow} p^{\prime}$,

- either there exists some $q^{\prime}$ such that $q(\stackrel{\tau}{\longrightarrow})^{+} q^{\prime}$ with $p^{\prime} \precsim R S q^{\prime}$,

- or $I^{*}\left(p^{\prime}\right)=A$ and $p^{\prime} \precsim_{R S} q$; and

$-I^{*}(q) \subseteq I^{*}(p)$.

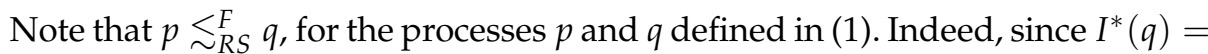
$A$, process $q$ can match the initial $\tau$-labelled transition from $p$ by remaining idle.

Proposition 8 (Behavioural characterization of $\bigsqcup_{R S}$ ). If $A$ is finite then $p \lesssim_{R S}^{F} q$ if, and only if, $p \sqsubseteq_{R S} q$, for all $p, q \in \mathrm{T}\left(A_{\tau}\right)$.

Ground-completeness In order to give a ground-complete axiomatization of the relation $\lesssim_{R S}$, we consider the equation

$$
\left(R S_{\Sigma}\right) \quad \tau\left(\sum_{a \in A} a x_{a}+y\right)=\sum_{a \in A} a x_{a}+y
$$

Proposition 9. The set of equations $E_{R S \leq}^{F c}=B W \cup\left\{R S_{\tau}, R S_{\Sigma}\right\}$, in which $R S_{\tau}$ is conditional, is sound and ground-complete for $\lesssim_{R S}^{F}$ over the language $\operatorname{BCCSP}\left(A_{\tau}\right)$.

Theorem 5. The set of equations

$$
E_{R S \leq}^{F}=B W \cup\left\{R S, \tau g, R S_{\Sigma}\right\}
$$

is sound and ground-complete for $\operatorname{BCCSP}\left(A_{\tau}\right)$ modulo $\lesssim_{R S}^{F}$.

We now proceed to offer (un)conditional axiomatizations of $\bar{\sim}_{R S}^{F}$, the kernel of the preorder $\lesssim_{R S}$.

Proposition 10. The set of equations $E_{R S}^{F c}=B W \cup\left\{R S E_{\tau}, R S_{\Sigma}\right\}$, in which $R S E_{\tau}$ is conditional, is sound and ground-complete for $\operatorname{BCCSP}\left(A_{\tau}\right)$ modulo $\bar{\sim}_{R S}^{F}$.

Theorem 6. The set of equations

$$
E_{R S^{=}}^{F}=B W \cup\left\{R S E, R S_{\tau e}, R S_{\Sigma}\right\}
$$

is sound and ground-complete for $\operatorname{BCCSP}\left(A_{\tau}\right)$ modulo $\bar{\sim}_{R S}^{F}$.

Remark 3. Since, in the case $|A|<\infty$, the preorder $\lesssim_{R S}^{F}$ is the largest precongruence included in $\precsim_{R S}$, all the axiomatizations above are also sound and groundcomplete for $\sqsubseteq_{R S}$ and its kernel, in this case. 


\begin{tabular}{|c|c|c|c|c|}
\hline $\begin{array}{c}\text { Weak Ready Simulation } \\
\text { Finite Equations }\end{array}$ & \multicolumn{2}{|c|}{ Ground-complete } & \multicolumn{2}{c|}{ Complete } \\
\cline { 2 - 5 } & Order & Equiv. & Order & Equiv. \\
\hline$|A|=\infty$ & $E_{R S \leq}$ & $E_{R S}=$ & $E_{R S \leq}$ & $E_{R S}=$ \\
\hline $1 \leq|A|<\infty$ & $E_{R S \leq}^{F}$ & $E_{R S}^{F}=$ & \multicolumn{2}{|c|}{ Do not exist } \\
\hline
\end{tabular}

Table 3. Axiomatizations for the largest (pre)congruence included in the weak ready simulation semantics

Nonexistence of finite complete axiomatizations We shall now prove that, if the set of actions $A$ is finite, then neither $\lesssim_{R S}^{F}$ nor its kernel afford a finite (in)equational axiomatization. The following proposition was shown in [15].

Proposition 11. For each $n \geq 0$, the equation

$$
a^{n} x+a^{n} \mathbf{0}+\sum_{b \in A} a^{n}(x+b)=a^{n} \mathbf{0}+\sum_{b \in A} a^{n}(x+b)
$$

is sound modulo ready simulation equivalence, and therefore modulo the kernel of $\lesssim_{R S}^{F}$.

The family of equations (2) plays a crucial role in the proof of Theorem 36 in [15], to the effect that the equational theory of ready simulation equivalence is not finitely based over $\operatorname{BCCSP}\left(A_{\tau}\right)$ when the set of actions is finite and contains at least two distinct actions. (In fact, as we showed in [2], ready simulation semantics is not finitely based, even when the set of actions is a singleton.)

Theorem 7. If $|A| \geq 1$ then the (in)equational theory of $\lesssim_{R S}^{F}$ over $\operatorname{BCCSP}\left(A_{\tau}\right)$ does not have a finite (in)equational basis. In particular, the following statements hold true.

1. No finite set of sound inequations over $\operatorname{BCCSP}\left(A_{\tau}\right)$ modulo $\lesssim_{R S}^{F}$ can prove all of the sound inequations in the family

$$
a^{n} x \leq a^{n} \mathbf{0}+\sum_{b \in A} a^{n}(x+b) \quad(n \geq 1) .
$$

2. No finite set of sound (in)equations over $\mathrm{BCCSP}\left(A_{\tau}\right)$ modulo $\lesssim_{R S}^{F}$ can prove all of the sound equations in the family (2).

Theorem 7 is a corollary of the following result. As usual, we consider processes up to strong bisimilarity.

Proposition 12. Assume that $|A| \geq 1$. Let $E$ be a collection of inequations whose elements are sound modulo ${\underset{\sim}{R S}}_{F}$ and have depth smaller than $n$. Suppose furthermore that the inequation $t \leq u$ is derivable from $E$ and that $u \lesssim_{R S}^{F} a^{n} \mathbf{0}+\sum_{b \in A} a^{n}(x+b)$. Then $t \stackrel{a^{n}}{\Longrightarrow} x$ implies $u \stackrel{a^{n}}{\Longrightarrow} x$. 
Corollary 2. If $1 \leq|A|<\infty$ then the collection of (in)equations in at most one variable that hold over $\operatorname{BCCSP}\left(A_{\tau}\right)$ modulo $\lesssim_{R S}$ does not have a finite (in)equational basis. Moreover, for each $n$, the collection of all sound (in)equations of depth at most $n$ cannot prove all the valid (in)equations in at most one variable that hold in weak ready simulation semantics over $\operatorname{BCCSP}\left(A_{\tau}\right)$.

\begin{tabular}{|c|ll|}
\hline \multicolumn{3}{|c|}{ Unconditional } \\
\hline$E_{R S \leq}=B W \cup\{R S, \tau g\}$ & $(R S)$ & $a x \leq a x+a y$ \\
$E_{R S=}=B W \cup\left\{R S E, R S_{\tau e}\right\}$ & $(\tau g)$ & $x \leq \tau x$ \\
$E_{R S \leq}^{F}=B W \cup\left\{R S, \tau g, R S_{\Sigma}\right\}$ & & $\alpha(b x+z+b y)=$ \\
$E_{R S=}^{F}=B W \cup$ & $\alpha(b x+z+b y)+\alpha(b x+z)$ \\
& $\left(R S S_{\tau e}\right)$ & $\alpha(x+\tau y)=$ \\
& \multicolumn{2}{|c|}{$\alpha\left(x+\tau S_{\tau e}, R S_{\Sigma}\right\}$} \\
& $\left(R S_{\Sigma}\right)$ & $\tau\left(\sum_{A} a x_{a}+y\right)=\sum_{A} a x_{a}+y$ \\
\hline \multicolumn{3}{|c|}{ Conditional } \\
\hline & $\left(R S_{\tau}\right)$ & $\left(I^{*}(x) \Leftrightarrow I^{*}(y)\right) \Rightarrow$ \\
$E_{R S \leq}^{c}=B W \cup\left\{R S_{\tau}\right\}$ & & $x \leq x+y$ \\
$E_{C S=}^{c}=B W \cup\left\{R S E_{\tau}\right\}$ & $\left(R S E_{\tau}\right)$ & $\left(I^{*}(x) \Leftrightarrow I^{*}(y)\right) \Rightarrow$ \\
& & $\alpha(x+y)=\alpha(x+y)+\alpha x$ \\
\hline
\end{tabular}

Table 4. Axioms for the largest (pre)congruence included in the weak ready simulation semantics

Tables 3 and 4 summarize the positive and negative results on the existence of finite axiomatizations for weak ready simulation semantics.

\section{Conclusion}

In this paper, we have offered a detailed study of the axiomatizability properties of the largest (pre)congruences over the language BCCSP induced by the 'weak' versions of the classic simulation and ready simulation preorders and equivalences. For each of these notions of behavioural semantics, we have presented results related to the (non)existence of finite (ground-)complete (in)equational axiomatizations. As in [15], the finite axiomatizability of the studied notions of semantics depends crucially on the cardinality of the set of observable actions. Following [17], we have also discussed ground-complete axiomatizations of those semantics using conditional (in)equations in some detail. In particular, we have shown how to prove ground-completeness results for (in)equational axiom systems from similar results for conditional axiomatizations in a fairly systematic fashion. 
The results presented in this article paint a rather complete picture of the axiomatic properties of the above-mentioned weak simulation semantics over BCCSP. We have also obtained similar results for the intermediate case of the weak complete simulation semantics, to be presented in a forthcoming paper. Moreover, in the cases in which the studied notions of semantics do not afford finite complete axiomatizations, it would be interesting to obtain infinite, but finitely described, complete axiomatizations. This is a topic that we leave for future research.

The results presented in this study complement those offered in, e.g., [7,37, $38,41]$, where notions of divergence-sensitive preorders based on variations on prebisimilarity $[26,32]$ or on the refusal simulation preorder have been given ground-complete inequational axiomatizations. They are just a first step in the study of the equational logic for notions of behavioural semantics in the extension of van Glabbeek's spectrum to behavioural semantics that abstract from internal steps in computation [22].

A natural avenue for future research is to investigate the equational logic of weak versions of semantics in van Glabbeek's spectrum that are based on notions of decorated traces. We have already started working on this topic and we plan to report on our results in a forthcoming article.

Following the developments in $[1,10,37]$, it would also be interesting to study rule formats for operational semantics that provide congruence formats for the semantics considered in this paper, and to give procedures for generating ground-complete axiomatizations for them for process languages in the given formats.

In [16], Chen, Fokkink and van Glabbeek have provided an extension to weak process semantics of the 'ready to preorder' procedure for generating axiomatizations of process equivalences from those of their underlying preorders, first studied in $[3,20]$. It would be worthwhile to study whether the scope of the algorithm presented in [16] can be extended to cover the case of the weak ready simulation congruence in Definition 4 and related semantics. The doctoral dissertation [13] also presents an algorithm to turn an axiomatization of a semantics for concrete processes into one for 'its induced weak semantics.' An extension of the scope of applicability of that algorithm would also be a significant advance on the state of the art in the study of axiomatizability results for process semantics over process algebras.

\section{References}

1. L. Aceto, B. Bloom, and F. W. Vaandrager. Turning sos rules into equations. Information and Compututation, 111(1):1-52, 1994.

2. L. Aceto, D. de Frutos Escrig, C. Gregorio-Rodríguez, and A. Ingólfsdóttir. Complete and ready simulation semantics are not finitely based over BCCSP, even with a singleton alphabet. Information Processing Letters, 111(9):408-413, 2011.

3. L. Aceto, W. Fokkink, and A. Ingólfsdóttir. Ready to preorder: get your BCCSP axiomatization for free! In Algebra and Coalgebra in Computer Science, Second Inter- 
national Conference, CALCO 2007, volume 4624 of Lecture Notes in Computer Science, pages 65-79. Springer, 2007.

4. L. Aceto, W. Fokkink, A. Ingólfsdóttir, and B. Luttik. Finite equational bases in process algebra: Results and open questions. In Processes, Terms and Cycles, volume 3838 of Lecture Notes in Computer Science, pages 338-367. Springer, 2005.

5. L. Aceto, W. Fokkink, A. Ingolfsdottir, and B. Luttik. A finite equational base for CCS with left merge and communication merge. ACM Trans. Comput. Log., 10(1), 2009.

6. L. Aceto, W. Fokkink, A. Ingólfsdóttir, and M. R. Mousavi. Lifting non-finite axiomatizability results to extensions of process algebras. Acta Informatica, 47(3):147-177, 2010.

7. L. Aceto and M. Hennessy. Termination, deadlock and divergence. Journal of the ACM, 39(1):147-187, 1992.

8. L. Aceto, A. Ingolfsdottir, B. Luttik, and P. van Tilburg. Finite equational bases for fragments of CCS with restriction and relabelling. In 5th IFIP International Conference on Theoretical Computer Science, volume 273 of IFIP, pages 317-332. Springer, 2008.

9. J. Baeten, T. Basten, and M. Reniers. Process Algebra: Equational Theories of Communicating Processes, volume 50 of Cambridge Tracts in Theoretical Computer Science. Cambridge University Press, November 2009.

10. J. C. M. Baeten and E. P. de Vink. Axiomatizing gsos with termination. Journal of Logic and Algebraic Programming, 60-61:323-351, 2004.

11. J. A. Bergstra and J. W. Klop. Process algebra for synchronous communication. Information and Control, 60(1-3):109-137, 1984.

12. B. Bloom, S. Istrail, and A. R. Meyer. Bisimulation can't be traced. Journal of the ACM, 42(1):232-268, 1995.

13. T. Chen. Clocks, Dice and Processes. PhD thesis, Centrum voor Wiskunde en Informatica (CWI), Vrije Universiteit Amsterdam, 2009.

14. T. Chen and W. Fokkink. On the axiomatizability of impossible futures: Preorder versus equivalence. In LICS, pages 156-165. IEEE Computer Society, 2008.

15. T. Chen, W. Fokkink, B. Luttik, and S. Nain. On finite alphabets and infinite bases. Information and Computation, 206(5):492-519, 2008.

16. T. Chen, W. Fokkink, and R. J. van Glabbeek. Ready to preorder: The case of weak process semantics. Information Processing Letters, 109(2):104-111, 2008.

17. D. de Frutos-Escrig, C. Gregorio-Rodríguez, and M. Palomino. On the unification of process semantics: Equational semantics. Electronic Notes in Theoretical Computer Science, 249:243-267, 2009.

18. D. de Frutos-Escrig and C. Gregorio-Rodríguez. Universal coinductive characterizations of process semantics. In 5th IFIP International Conference on Theoretical Computer Science, volume 273 of IFIP, pages 397-412. Springer, 2008.

19. D. de Frutos-Escrig and C. Gregorio-Rodríguez. (Bi)simulations up-to characterise process semantics. Information and Computation, 207(2):146-170, 2009.

20. D. de Frutos-Escrig, C. Gregorio-Rodríguez, and M. Palomino. Ready to preorder: an algebraic and general proof. Journal of Logic and Algebraic Programming, 78(7):539$551,2009$.

21. R. De Nicola and M. Hennessy. Testing equivalences for processes. Theoretical Computer Science, 34:83-133, 1984.

22. R. v. Glabbeek. The linear time - branching time spectrum II. In CONCUR'93 - Concurrency Theory, 5th International Conference, volume 715 of Lecture Notes in Computer Science, pages 66-81. Springer, 1993. 
23. R. v. Glabbeek. The linear time - branching time spectrum I; the semantics of concrete, sequential processes. In Handbook of Process Algebra, chapter 1, pages 3-99. Elsevier, 2001.

24. R. v. Glabbeek and P. Weijland. Branching time and abstraction in bisimulation semantics. Journal of the ACM, 43(3):555-600, 1996.

25. J. F. Groote. A new strategy for proving omega-completeness applied to process algebra. In CONCUR '90, Theories of Concurrency: Unification and Extension, volume 458 of Lecture Notes in Computer Science, pages 314-331. Springer, 1990.

26. M. Hennessy. A term model for synchronous processes. Informationa and Control, 51(1):58-75, 1981.

27. M. Hennessy and R. Milner. Algebraic laws for nondeterminism and concurrency. Journal of the ACM, 32:137-161, 1985.

28. C. Hoare. Communicating Sequential Processes. Prentice Hall, 1985.

29. K. G. Larsen and A. Skou. Bisimulation through probabilistic testing. Information and Computation, 94(1):1-28, 1991.

30. G. Lüttgen and W. Vogler. Ready simulation for concurrency: It's logical! Inf. Comput., 208(7):845-867, 2010.

31. R. Milner. An algebraic definition of simulation between programs. In Proceedings 2nd Joint Conference on Artificial Intelligence, pages 481-489. BCS, 1971. Also available as Report No. CS-205, Computer Science Department, Stanford University.

32. R. Milner. A modal characterisation of observable machine behaviour. In E. Astesiano and C. Böhm, editors, CAAP '81: Trees in Algebra and Programming, 6th Colloquium, volume 112 of Lecture Notes in Computer Science, pages 25-34. Springer, 1981.

33. R. Milner. Communication and Concurrency. Prentice Hall, 1989.

34. R. Milner. A complete axiomatisation for observational congruence of finite-state behaviors. Information and Computation, 81(2):227-247, 1989.

35. F. Moller. Axioms for Concurrency. PhD thesis, Report CST-59-89, Department of Computer Science, University of Edinburgh, 1989.

36. G. D. Plotkin. A structural approach to operational semantics. Journal of Logic and Algebraic Programming, 60-61:17-139, 2004.

37. I. Ulidowski. Axiomatisations of weak equivalences for De Simone languages. In CONCUR '95: Concurrency Theory, 6th International Conference, volume 962 of Lecture Notes in Computer Science, pages 219-233. Springer, 1995.

38. I. Ulidowski. Refusal simulation and interactive games. In Algebraic Methodology and Software Technology, 9th International Conference, AMAST 2002, Lecture Notes in Computer Science, pages 208-222. Springer, 2002.

39. R. J. van Glabbeek. A characterisation of weak bisimulation congruence. In Processes, Terms and Cycles: Steps on the Road to Infinity, volume 3838 of Lecture Notes in Computer Science, pages 26-39. Springer, 2005.

40. M. Voorhoeve and S. Mauw. Impossible futures and determinism. Information Processing Letters, 80(1):51-58, 2001.

41. D. Walker. Bisimulation and divergence. Information and Computation, 85(2):202-241, 1990. 